\title{
DIAGNOSTIC ACCURACY OF MRI IN DIFFERENTIATING NON-MUSCLE INVASIVE FROM MUSCLE INVASIVE BLADDER CARCINOMA TAKING HISTOPATHOLOGICAL STAGING AS A STANDARD.
}

\author{
Syed Muhammad Faiq ${ }^{1}$, Khujasta Mehtab $^{1}$,Nazish $\mathrm{Naz}^{2}, \mathrm{Fatima} \mathrm{Batool}^{3}$, Kiran Nazi $^{3}$, Anwar Naqvi ${ }^{1}$ \& Adeeb ul Hassan \\ Sindh Institute of Urology \& Transplantation \\ Corresponding Author Email: sfaiq69@ hotmail.com
}

\section{ABSTRACT}

To Determine Diagnostic accuracy Of MRI in differentiating non-muscle invasive from muscle invasive bladder carcinoma taking Histopathological staging as a standard. The data of 53 patients with bladder tumor who previously confirmed by cystoscopy were examined at $1.5 \mathrm{~T}$, images were obtained and analyzed by radiologist without knowledge of the tumor stage. T2-weighted spin-echo or turbo-spin-echo, and dynamic Ti-weighted fast gradient-echo sequences were used. Histopathological grading served as standard of reference. Compared with the results submitted from the histopathology unit and using more detailed TNM classification, the sensitivity, specificity, and percent accuracy of MRI was $91 \%, 86 \%$, $85 \%$ respectively. This data confirms the under-staging of the bladder tumor invasion done histopathologically while MR is a promising, non-invasive technique for the detection of bladder lesions with a high diagnostic performance.

\section{KEYWORDS}

Bladder cancer, Transitional cell carcinoma, Magnetic resonance imaging, staging, Tumor node metastasis

\section{INTRODUCTION}

Carcinoma of the urinary bladder is one of the most common malignant tumors of the urinary tract in men and women and represents $2 \%$ of all malignancies. Almost all bladder cancers originate in the urothelium which is muscular layer in the bladder (Barentsz, 1993).

Bladder tumors are grouped in several types and it can affect treatment options, because different types can respond differently to radiation and chemotherapy (Wein, 2007)

\section{Causes of bladder cancer}

Certain factors make bladder cancer more likely.

- Smoking triples risk of bladder cancer. Passive smoking may also increase risk.

- Exposure to certain industrial chemicals (for example, those used in printing and textiles, gas and tar manufacturing, iron and aluminum-processing industries).
- A long-term infection with the tropical disease, schistosomiasis.

- A long-term or repeated bladder infection.( Van Rhijn, 2005)

\section{Types of bladder tumors}

Bladder tumors are grouped into several types by how their cells look under a microscope.This is because different types can respond differently to treatments such as radiation and chemotherapy (Jacobs, 2010)

The main types of cancers that affect the bladder are:

- Transitional cell carcinoma (also called urothelial carcinoma)

- Squamous cell carcinoma

- Adenocarcinoma

- Small cell carcinoma

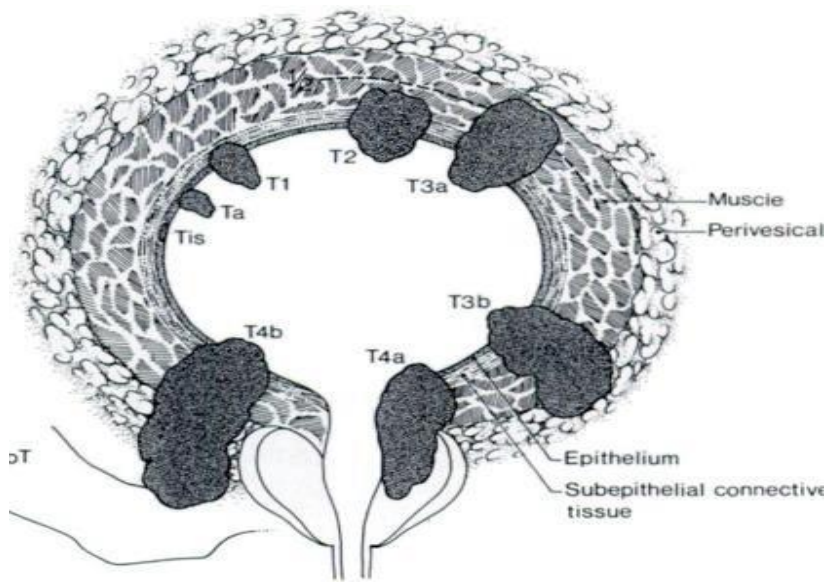

Fig.1- Depth of penetration according to TNM system of staging bladder carcinoma.

Grade: These tumors are divided into grades based on how abnormal cells look under the microscope. If the cells appear more like normal cells, the cancer is of low grade. If cells look more abnormal, this is High grade cancer. 


\section{Invasiveness:}

If the cancer stays in the inner layer of cells, it is called noninvasive. Bladder cancer described as superficial are non-muscle invasive. If the cancer grows into the lamina propria deeper into the muscle layer, it is called invasive. Invasive cancers are more likely to spread (Van Rhijn, 2005).

\section{AJCC TNM staging system for bladder cancer}

A staging system is a standard way to describe the extent of cancer spread for the members of the cancer care team.

The staging system most often used for bladder cancer is that of the American Joint Committee on Cancer (AJCC). This is also called the TNM system.

The TNM staging system classifies cancers based on 3 key pieces of information;

The letter $\mathrm{N}$ is followed by a number from 0 to 3 to indicate cancer has spread to lymph nodes near the bladder.

The letter $\mathrm{M}$ is followed by 0 or 1 to indicate whether cancer has spread (metastasized) or not to distant sites.

\section{T categories for bladder cancer}

The $\mathrm{T}$ category describes the main tumor.

TX: Main tumor can't be assessed due to lack of information T0: No evidence of a primary tumor

Ta: Non-invasive papillary carcinoma

Tis: Non-Invasive flat carcinoma (in situ)

T1: The tumor has grown from the layer of cells lining the bladder into the connective tissues below. It has not grown into the muscle layer of the bladder.

T2: The tumor has grown into the muscle layer.

T2a: The tumor has grown to the inner half of the muscle layer.

T2b: The tumor has grown into the outer half of the muscle layer.

T3: The tumor has grown to the muscle layer of the bladder and fatty tissue that surrounds it.

T3a: The tumor's spread to fatty tissue on the outside of the bladder can only be seen by using a microscope.

T3b: The tumor's spread to fatty tissue on the outside of the bladder is large enough to be seen on imaging tests or to be seen or felt by the surgeon.

T4: The tumor has spread beyond the fatty tissue and into nearby organs.

T4a: The tumor has spread to stoma of prostate or to the uterus/vagina

T4b: The tumor spread to the pelvic wall or abdominal wall.

Bladder carcinoma affect many organs at the same time. If tumor is found, the letter $\mathbf{m}$ is added to the appropriate $\mathbf{T}$ category.

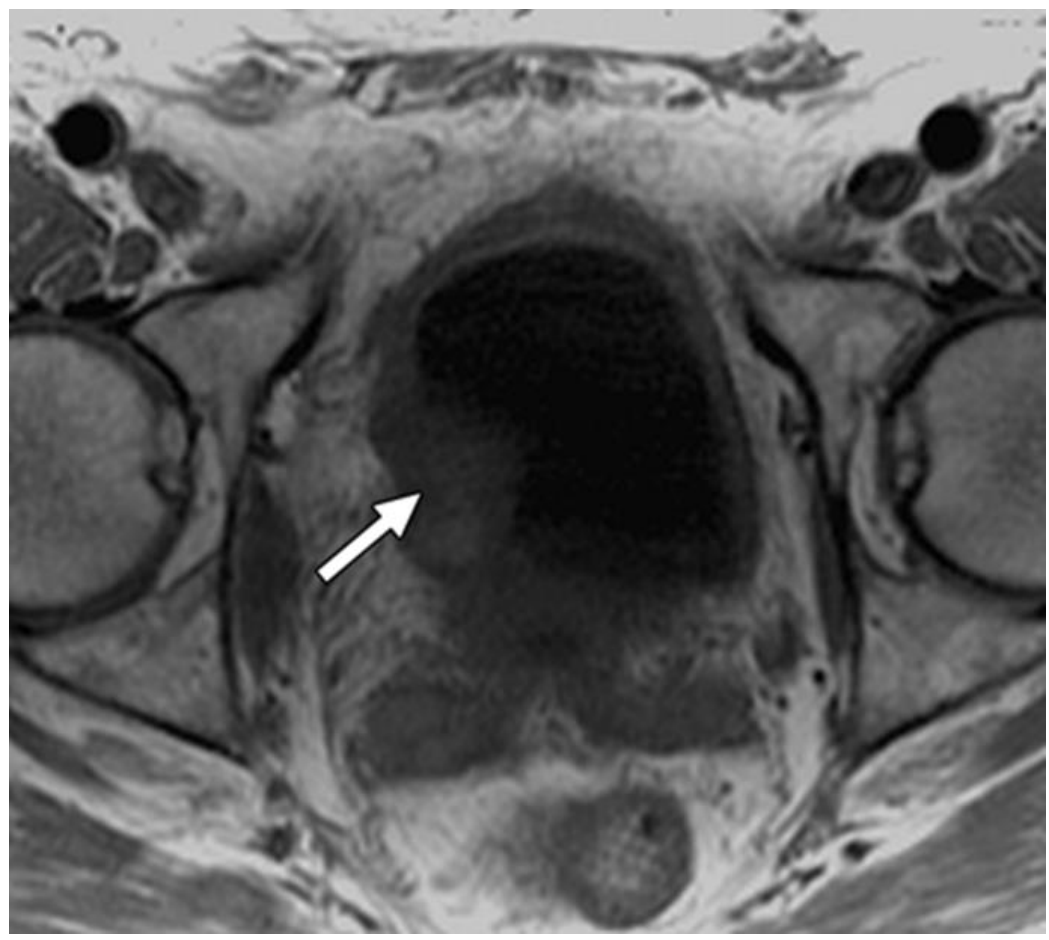

T1 weighted image showing decreased signal intensity of bladder wall (arrow showing less intense tumor) 


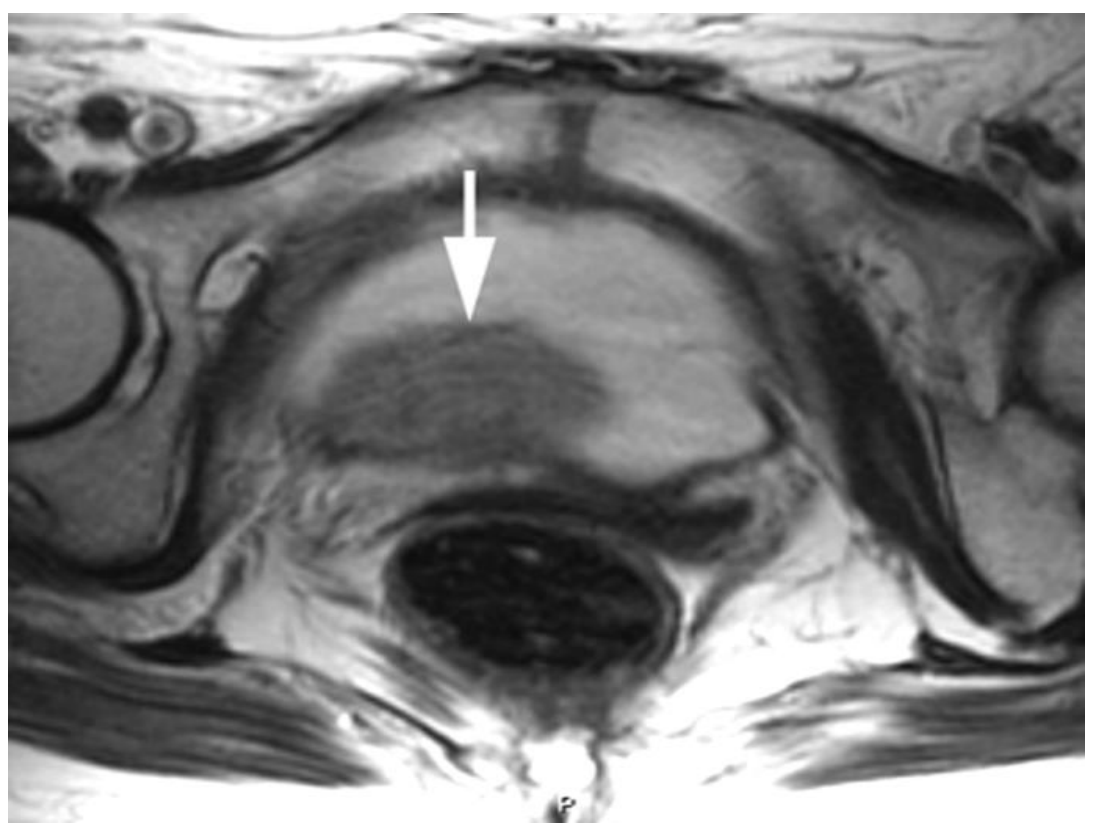

T2 weighted image of TCC bladder showing signals at the base of the bladder.

For differentiation between muscular invasion (stage T3a) and invasion into the perivesical fat (stage T3b), especially in tumors at the bladder dome or base, MR imaging is superior. In deeply infiltrative tumors (stages $3 \mathrm{~b}, \mathrm{~T} 4 \mathrm{a}$, and $\mathrm{T} 4 \mathrm{~b}$ ). This distinction cannot be made with clinical staging, CT, or intravesical sonography (Reiser, 2007).

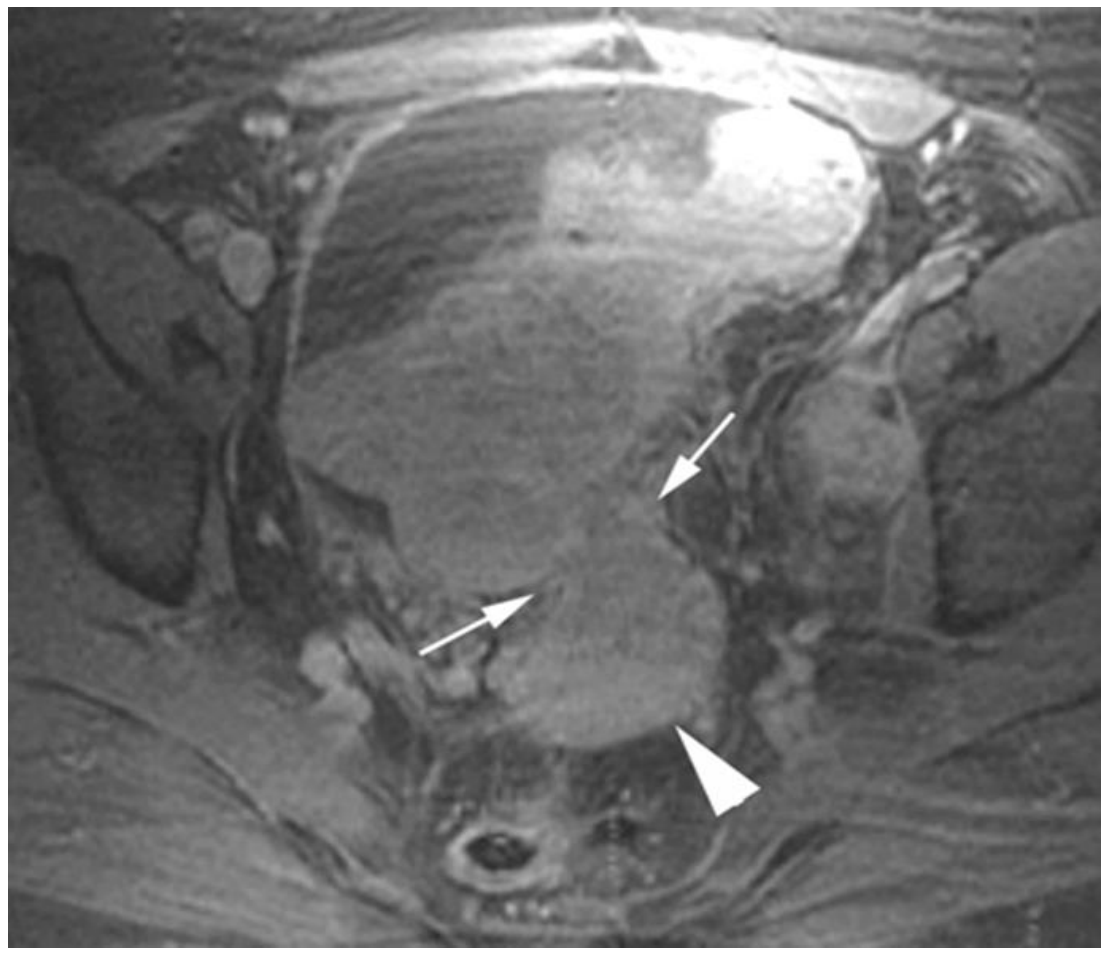

Contrast enhanced T1 weighted image of TCC bladder T4 stage (arrow shows invasion of tumor in adjacent viscera)

\section{Role of MRI}

Magnetic Resonance Imaging provides non invasive tool that is unrelated to radioactivity, to investigate the internal anatomy and the physiology. Magnetic resonance is a very low energy phenomenon, involving the absorption and emission of radiofrequency (RF) waves. The most important nuclei for magnetic resonance imaging (MRI) are hydrogen because it is present throughout the body in water and fat. It is based on the 
interaction between an applied magnetic field and a particle that possess spin (Husband, 1989).

MRI is an effective technique for evaluating full range of bladder diseases (Prasad, 2006). It is mainly used to stage known bladder cancer detect tumor recurrence, and evaluate for secondary invasion of bladder by gynecologic, prostatic or rectal neoplasm. In addition the multiplanar capabilities of MRI may provide important information in selected patients with congenital anomalies or inflammatory diseases (Lucas, 2006). MRI is superior at resolution of local invasion. Staging of Transitional cell carcinoma is the most common indication for bladder MRI investigation with image acquisitions in multiple planes and concurrent use of phased array multi coil. Use of MRI may provide dynamic information, lymph node specific contrast agents have been investigated that permit differentiation of lymph node malignant involvement versus hyperplasic enlargement thus the staging of bladder carcinoma is improved(Husband, 1989). MR imaging can detect tumor involvement of parenchyma and distant metastases. It therefore offers an staging modality and has been shown to stage TCC (Watson, 1993).

MRI examinations of the urinary bladder have most often been performed at a magnetic field strength of $1.5 \mathrm{~T}$. imaging protocols based on $\mathrm{T} 1$ weighted and $\mathrm{T} 2$ weighted images. The introduction of turbo or fast spin echo (TSE or FSE) has made T2-weighted MR imaging considerably faster.

\section{MATERIAL \& METHOD}

Retrospective study done in Department of Radiology, Sindh institute of urology and transplantation. (Tertiary care teaching Hospital).

\section{Sample size:}

53 patients underwent MR pelvis imaging because of suspected Transitional cell carcinoma of bladder and these patients, including men and women 17-55 years old (mean age, 36 years), formed the study population.

\section{Sample Selection}

\section{Inclusion criteria}

All patients of either gender and of age between 25-85 years who have an abnormal growth seen arising from bladder wall on cystoscopy.

\section{Exclusion Criteria}

Prior to pelvic radiation for Ca bladder

Pregnant and lactating patients

Patients who can't tolerate being in MR for the duration of study Patients with causes of hematuria

\section{Sampling Technique}

MR imaging was performed with a 1.5-T MR unit (Sigma Horizon, General Electric Medical Systems) with body phased array coil. Each patient underwent imaging with three MR pelvis techniques: $\mathrm{T} 1$ weighted images, $\mathrm{T} 2$ weighted images, Coronal stir with and without IV contrast.

\section{MR sequences and parameters}

T1 weighted Images:

T1 weighted SE sequence TR (400-800), TE (15-30)

T2 weighted sequence:

T2 weighted Se sequence TR (1600-2000), TE (75-100)

Short-TI inversion recovery (STIR) sequence:

A short-TI inversion recovery (STIR) sequence TR (2000), TE 60-75, T1 150-275.

\section{Data Analysis}

Sensitivity, specificity, positive and negative predictive values will be calculated using formula. Radiologically assigned $\mathrm{T}$ stage will be correlated with pathologically assigned stage determined $\mathrm{T}$ stage using Spearmen rank correlation test. Patient with radio logically negative tumor will be assigned stage $\mathrm{T} 0$ for statistical correlation. When multiple tumor are present, highest T Stage will represent tumor stage. In addition, data will be regrouped to evaluate accuracy of MRI in distinguishing superficial $(\leq \mathrm{T} 1)$ from invasive $(\geq \mathrm{T} 2)$.

\section{RESULTS}

Around 53 patients were included in this study, whose ages ranged from 30 years -80 years with mean age of 55 years. The male to female ratio was $5: 1$ (Fig. 1)

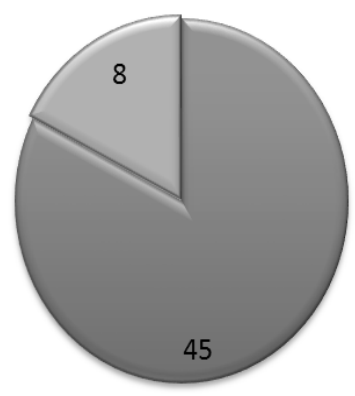

$\square$ Male $\square$ Female

Fig.1 Gender Distribution of Male and Female with Ca Bladder 
Hematuria was present in 45 cases. Out of the 53 patients with urinary bladder carcinoma 36 showed muscle invasive bladder carcinoma. Of these $36(68 \%)$ were reported as presence of muscle invasive bladder carcinomas (True $+\mathrm{ve}$ ) and $11(15 \%)$ were reported non-muscle invasive bladder carcinomas (True -ve) (fig. 2)

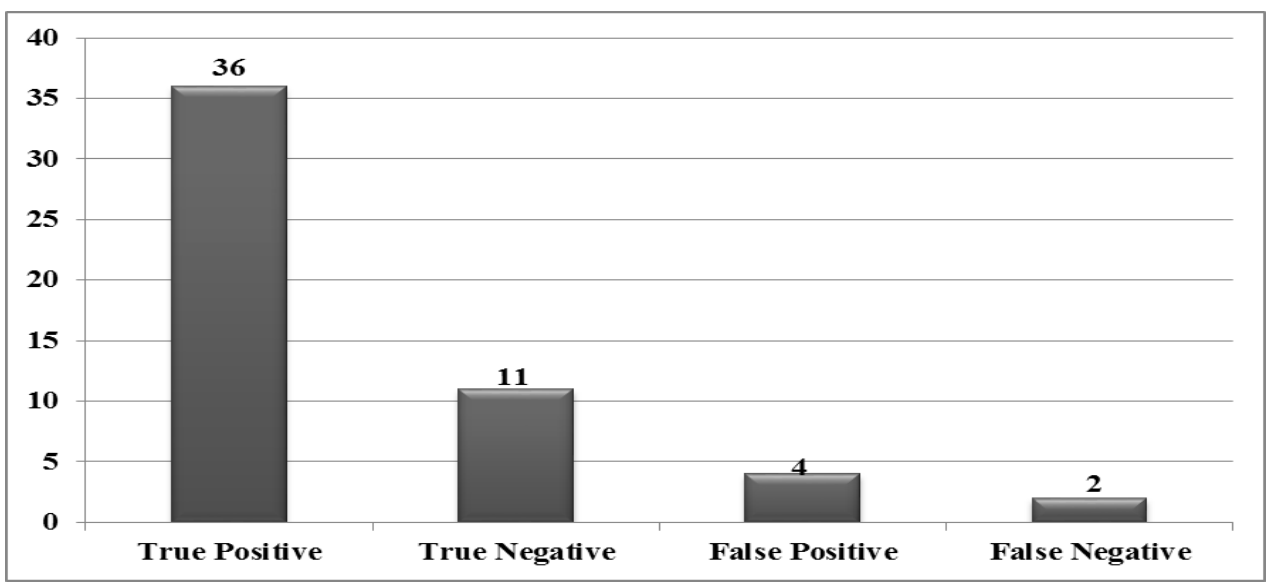

Fig.2: Distribution of Positive and Negative results of Ca Bladder on MRI vs. Histopathology

MRI was False-positive in 4(40\%) patients and False-negative (understaged) in $2(5 \%)$. When compared with the results submitted from the histopathology unit and using more detailed
TNM classification, the sensitivity, specificity, and percent accuracy of MRI was $91 \%, 86 \%, 85 \%$ respectively (Table 1 ).

\begin{tabular}{|l|l|l|l|}
\hline $\begin{array}{l}\text { Superficial v/s Organ Confined } \\
\text { Muscle-invasive }\end{array}$ & Sensitivity & Specificity & Accuracy \\
\cline { 2 - 4 } & $91 \%$ & $86 \%$ & $85 \%$ \\
\hline
\end{tabular}

Table: 1 Measurements of the values of MRI for Superficial v/s muscle invasive organ confined bladder carcinoma

\section{DISCUSSION}

MR imaging and clinical staging is mandatory for the staging of urinary bladder carcinoma. The treatment and prognosis of urinary bladder carcinoma is largely determined by the depth of the tumor growth and its extent. Bladder-saving treatment is used for superficial tumors (stages Ta-T1), whereas for stage T2-T3b tumors, radical cystectomy is performed. The treatment for stage $\mathrm{T} 4 \mathrm{a}$ and $\mathrm{T} 4 \mathrm{~b}$ tumors and for metastatic disease is usually chemotherapy or palliative radiation and MR imaging is superior in determination of local tumor growth as well as metastases, with the combination of sequences and selective contrast agents it is possible to detect the tumor in early stages. Limitation of the clinical staging for the diagnosis of bladder tumor is the determination of extent of tumor growth in the muscle layer of the bladder wall (stages T2 and >T2), however the role of MR in bladder tumor is continue to evolve (Sharma, 2011). MRI has its own limitations. There are number of factors which can be patient related or MRI related. The most important are motion artifacts and the degree of bladder distension (Zhang, 2007).

Optimal bladder distension is very important. In a bladder that is not sufficiently distended, the muscle is thickened, which makes difficult in visualizing small tumors. In other case if the bladder becomes too distended, the patient becomes restless. Because of the over stretching of the muscle layer, flat tumors can be missed. Voluntary motion artifacts can be reduced by making the patient feel ease in case of claustrophobic patients, sedatives may be effective. Involuntary motion artifacts are caused by respiration, intestinal peristalsis and bladder motion (Jacobs, 2010). Multiplanar imaging allows better visualization of the bladder dome, trigone, and adjacent structures such as the prostate and seminal vesicles. Its excellent resolution and high soft-tissue contrast can even be enhanced by using paramagnetic contrast agents. In lower-stage tumors stages Ta, T1, and T2), MR imaging has limitations. Biopsy is the best method for clinical staging of superficial tumors. After the cystoscopy detection of bladder tumor staging of the urinary bladder tumor should start with technically improved MR imaging followed by clinical staging. Accuracy improves with Phased array coils, Gadolinium enhancement and pulse sequences.

\section{CONCLUSION}

This data confirms the under-staging of the bladder tumor invasion done histopathologically while MR is a promising, non-invasive technique for the detection of bladder lesions with a high diagnostic performance.

\section{REFERENCES}

- $\quad$ Barentsz, J. O., Ruijs, S. H., \& Strijk, S. P. (1993). The role of MR imaging in carcinoma of the urinary bladder. American journal of roentgenology, 160(5), 937-947.

- Husband, J. E., Olliff, J. F., Williams, M. P., Heron, C. W., \& Cherryman, G. R. (1989). Bladder cancer: staging with CT and MR imaging. Radiology, 173(2), 435-440. 
- Jacobs, B. L., Lee, C. T., \& Montie, J. E. (2010). Bladder cancer in 2010: how far have we come?. CA: a cancer journal for clinicians, 60(4), 244-272.

- $\quad$ Lucas, L. W., \& Lockhart, M. E. (2006). Abdominal-Pelvic MRI. American Journal of Roentgenology, 187(4), 439-445.

- Prasad, P. V. (Ed.). (2006). Magnetic resonance imaging: methods and biologic applications. Springer.

- $\quad$ Reiser, M. F., Semmler, W., \& Hricak, H. (2007). Magnetic resonance tomography. Springer.

- Sharma, V. (2011). MR Imaging of the Abdomen and Pelvis. Academic Radiology, 18(2), 264-265.

- Van Rhijn, B. W., Van der Poel, H. G., \& van Der Kwast, T. H. (2005). Urine markers for bladder cancer surveillance: a systematic review. European urology, 47(6), 736-748.

- Watson, J. D., Myers, R., Frackowiak, R. S., Hajnal, J. V., Woods, R. P., Mazziotta, J. C., ... \& Zeki, S. (1993). Area V5 of the human brain: evidence from a combined study using positron emission tomography and magnetic resonance imaging. Cerebral Cortex, 3(2), 79-94.

- Wein, A. J., Kavoussi, L. R., Novick, A. C., Partin, A. W., \& Peters, C. A. (2007). Campbell-Walsh Urology edition. JAMA, 298(18), 2201-2202.

- Zhang, J., Lefkowitz, R. A., \& Bach, A. (2007). Imaging of kidney cancer. Radiologic Clinics of North America, 45(1), 119-147. 